\title{
NOTES
}

\section{THOMPSON: QUINTETS WITH VARIATIONS}

\begin{abstract}
The Editors
Ken Thompson, whose persistence seems boundless, has given the Chess World a third Volume of his CDROMs, a medium which becomes increasingly accessible to the serious information techno-amateur. It has been truly said that research is endless and that good research is not unaptly known for raising more questions than it solves. So with the five men contending for a win. Roughly a year ago, the indefatigable Ken could claim with some justice that his work had been completed with the second Volume (The Editors, 1992). Alas, completion has not even been reached with a third Volume, although Ken Thompson expressively wrote: "There are almost no endgames $(5$ pce) left so this will probably be the last." None of the Volumes issued so far treats the KBNKP endgame for the various pawn files. This shortcoming, we have no doubt, will be removed in due time.
\end{abstract}

What is more serious is that FIDE now has declared those games to be statutorily drawn in which there has been no capture or pawn move for the duration of 50 moves. This forces us to reclassify some results. We note that all games which have an over-50-moves maximin in Table 1 involve a Pawn, and so do many in Volumes 1 and 2.

Consider an endgame with a maximin length of over 50 moves. In order now to determine whether it is FIDE-drawn it does no longer suffice to exhibit a witness in which the FIDE-conditions for drawing obtain. In such cases, it becomes necessary to explore not only the equi-optimal but also mildly sub-optimal moves in order to be able to state whether, say by a clever interchange of moves, the Pawn in question can or cannot be made to move within the first 50 moves, in which case, as we understand the rules, a new period of grace is entered upon (cf. Dekker et al., 1990).

Some purists will complain that the metric applied so far almost universally in the endgame-database world has been distance-to-conversion. These purists would greatly welcome the ultimate metric, application of which imposes considerable rewriting of programs for their construction. We feel sure nobody's respect for Thompson is diminished when we note he has not, so far, feel compelled to change metrics in midstream. We mention in passing that some of Stiller's (1989) results, published in this Journal, have now been confirmed by Thompson, where the latter has the advantage of having gone public to a degree. When confronting the outcomes in Dekker et al. (1987) with Thompson's, a minor difference should be kept in mind. The former's KNNKP(h)'s 115 moves (also on ICCA's posters) include the final mating move after Black has promoted his Pawn, whereas Thompson's 114 do not: a difference without a distinction.

We find, as Editors, that the work is never finished: the ultimate metric is still calling for constructors; new research questions are posed by FIDE's new ruling (cf. Van den Herik and Herschberg, 1993).

We can do no better justice to this giant undertaking than by listing sample results. For the endgames in Volume 3 we identify them, state their maximin, give a sample maximin position, indicate the side to move, note duplications, and code the conversion where applicable as follows: 1=mate (without conversion); 2=White promotes; $3=$ White captures; 4=Black promotes; 5=Black captures (not in the Table 1). The maximins indicate the number of moves White must play to arrive at conversion, even if Black effects the actual conversion. Consequently, e.g., 1... b2 2. Qf3 b1Q has a maximin of 1. 


\begin{tabular}{|c|c|c|c|c|}
\hline Endgame & Maximin & A maximin position & To move & $\begin{array}{c}\text { Conversion } \\
\text { code }\end{array}$ \\
\hline $\mathrm{KNNKB}^{2)}$ & 4 & (wnc8b5, wkd8, bbb7, bka8) & $\mathrm{w}$ & 1 \\
\hline $\mathrm{KNNKN}^{1)}$ & 7 & (wnc8c7, wkd7, bnh1, bkb8) & $\mathrm{w}$ & 1 \\
\hline KNNKP(a) & 114 & (wnb8f4, wka2, bpa6, bke4) & $\mathrm{w}$ & 4 \\
\hline KNNKP(b) & 75 & (wnc6f6, wka8, bpb7, bkc8) & $\mathbf{w}$ & 1 \\
\hline KNNKP(c) & 83 & (wnh4a2, wka8, bpc7, bkc8) & b & 4 \\
\hline KNNKP(d) & 97 & (wna4b1, wkg8, bpd7, bkd6) & b & 4 \\
\hline KNNKQ & 1 & (wnd7e6, wka8, bqe8, bkc8) & $\mathrm{w}$ & 1 \\
\hline KNNKR & 3 & (wne8d5, wkc8, bra6, bka8) & w & 1 \\
\hline $\mathrm{KRP}(\mathrm{a}) \mathrm{KB}$ & 47 & (wpa3, wrg6, wkh4, bbf8, bkd3) & b & 1 \\
\hline $\mathrm{KRP}(\mathrm{b}) \mathrm{KB}$ & 45 & (wpb3, wra5, wkg1, bbf6, bke4) & b & 1 \\
\hline $\mathrm{KRP}(\mathrm{c}) \mathrm{KB}$ & 43 & (wpc2, wrc1, wkh7, bba4, bka7) & b & 1 \\
\hline $\mathrm{KRP}(\mathrm{d}) \mathrm{KB}$ & 62 & (wpd3, wra6, wka1, bbg2, bka4) & b & 2 \\
\hline KQKRP(a) & 54 & (wqh4, wkh6, bpa2, brb5, bkf1) & b & 1 \\
\hline KQKRP(b) & 78 & (wqa8, wkc1, bpb7, brg4, bka6) & b & 3 \\
\hline KQKRP(c) & 79 & (whh7, wkh6, bpc4, brc5, bkb2) & w & 3 \\
\hline KQKRP(d) & 51 & (wqb8, wkh8, bpd4, brf1, bke4) & b & 3 \\
\hline KQKQR & 9 & (wqc1, wkc6, brg7, bqe2, bkb8) & $\mathrm{w}$ & 3 \\
\hline $\mathrm{KQKRB}^{1)}$ & 42 & (wqg8, wkc8, bba4, brc5, bkh6) & $\mathrm{w}$ & 3 \\
\hline $\mathrm{KQKRN}^{1)}$ & 46 & (wqe8, wkc8, bnb8, brb2, bke3) & b & 3 \\
\hline $\mathrm{KQKRR}^{1)}$ & 20 & (wqh3, wkd7, brg8a2, bkd3) & b & 3 \\
\hline KQRKB & 5 & (wrd8, wqh8, wka8, bbh7, bkf7) & b & 3 \\
\hline KRKBB & 9 & (wrg5, wkd8, bbe4g2, bka8) & w & 1 \\
\hline KRKBN & 13 & (wrb3, wkc8, bnc7, bba4, bka8) & $\mathrm{w}$ & 3 \\
\hline KRKNN & 11 & (wrb8, wkc7, bnc5g1, bka6) & $\mathrm{w}$ & 3 \\
\hline KRKBP(a) & 21 & (wrb1, wke5, bpa7, bbf3, bkh4) & w & 3 \\
\hline KRKBP(b) & 20 & (wrc2, wkd2, bpb5, bbf $2, \mathrm{bka} 1)$ & $\mathbf{w}$ & 1 \\
\hline $\mathrm{KRKBP}(\mathrm{c})$ & 18 & (wrg1, wkf1, bpc6, bba4, bkh1) & b & 3 \\
\hline KRKBP(d) & 15 & (wrb8, wka5, bpd7, bbb2, bka1) & w & 3 \\
\hline KRKNP(a) & 42 & (wra4, wka5, bpa7, bnf1, bka8) & w & 3 \\
\hline KRKNP(b) & 34 & (wra3, wkc7, bpb7, bnf8, bkh1) & $\mathrm{w}$ & 3 \\
\hline KRKNP(c) & 36 & (wre7, wkd7, bpc6, bnh4, bkh8) & w & 3 \\
\hline KRKNP(d) & 26 & (wra3, wke7, bpd5, bnf5, bkg8) & $\mathbf{w}$ & 3 \\
\hline KRKQB & 2 & (wrb5, wkc8, bbe8, bqd6, bka8) & w & 1 \\
\hline KRKQN & 3 & (wrb8, wkb7, bna8, bqg1, bkh5) & w & 3 \\
\hline KRKRB & 4 & (wrb7, wkc7, bbe2, brd8, bka8) & w & 1 \\
\hline KRKRN & 5 & (wrc4, wkd8, bng2, brd5, bka8) & w & 1 \\
\hline $\mathrm{KRBKB}^{2)}$ & 25 & (wba7, wrb6, wka8, bba4, bkc8) & b & 1 \\
\hline $\mathrm{KRNKB}^{2)}$ & 25 & (wnb1, wrc1, wkd8, bbh6, bka3) & b & 1 \\
\hline KRRKB & 10 & (wrc6f3, wka8, bbf7, bke4) & b & 3 \\
\hline
\end{tabular}

Table 1: Contents of Thompson's Volume 3 summarized.

1) also on Volume 1

2) also on Volume 2

\section{A Few Examples}

We also provide a sample of maximin games, again strictly following Ken Thompson, i.e., following the metric of distance-to-conversion. Thompson also supplies complete paths even after conversion, which we include; this does not constitute application of the ultimate metric. As usual, equi-optimal moves are in parentheses. 
KNNKP(d)

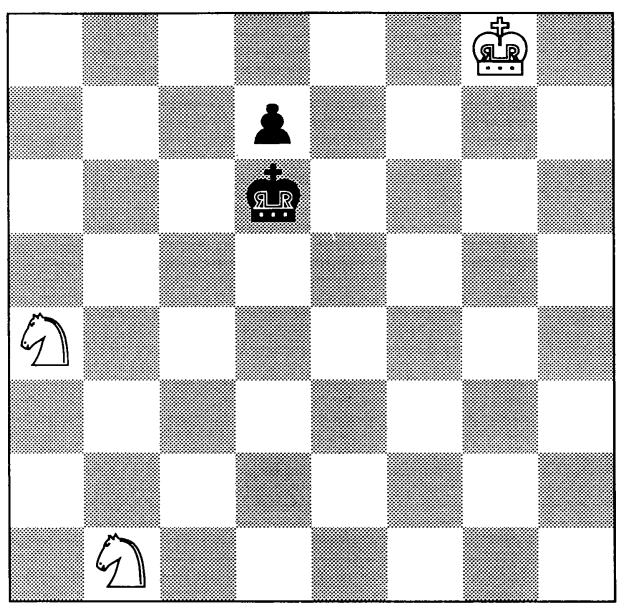

1. ... Kd5 2. Nbc3+ Kc4 3. Nd1 Kd3 4. Nc5+ Kd4 5. $\mathrm{Nb3}+\mathrm{Kd} 3$ 6. Nf2+ Ke3 7. Ng4+ Kf4 8. Nh6 Ke4 9. Nc1 Kf3 (Kf4 Ke3) 10. Nf5 (Nf7) Ke4 11. Nd6+ Kd5 12. $\mathrm{Ne} 8 \mathrm{Kc} 4(\mathrm{Ke} 4 \mathrm{Kc} 5 \mathrm{Kd} 4)$ 13. Nf6 Kb4 (Kd4 Kc3) 14. Kf7 (Kh7 Kf8 Kg7 Nh5 Ng4 Ne4 Nd5+) Kc3 15. Nd5+ Kd4 16. Nf4 (Nb4) Ke5 (Ke3 Ke4) 17. Ng2 (Nfd3+ Ncd3+) d6 (d5 Kd4 Kd5 Kf5 Ke4) 18. Ne1 (Nb3) d5 (Kd4) 19. Ncd3+ (Nb3) Kd4 (Kf5 Ke4) 20. Ke6 (Kf6) Kc4 (Ke4) 21. Kf5 (Ke5) Kc3 22. Kg4 (Ke5 Kf4) Kd2 23. Kf3 Kd1 24. Ke3 (Kf2) d4+ 25. Kf3 Kd2 26. Kf2 Kd1 27. Nf3 Kc2 28. Ke2 Kc3 29. Ng5 (Nfe1 Nd2 Nfe5) Kc4 (Kc2) 30. Ne4 (Ne6 Kd2) Kb3 31. Nec5+ (Kd1 Kd2) Kc4 32. Kd1 (Kd2) Kb5 (Kd5) 33. Kc2 Kc6 (Kb6) 34. Kb3 (Kb2) Kd5 (Kc7) 35. Kb4 Kc6 36. Ka5 Kd6 37. Kb6 Kd5 38. Kb5 Kd6 39. Kc4 Kc7 (Ke7 Kc6) 40. Kd5 Kb6 41. Ne4 Kc7 42. Ke5 Kd7 43. Kf6 Kc7 44. Ke7 Kc6 45. Ke6 Kc7 46. Kd5 Kd7 47. Nf6+ Ke7 48. Ke5 Kf7 49. Nd5 Kg6 50. N5b4 (Kf4)

Kg5 51. Nc2 Kg6 52. Ke6 Kg7 53. Na3 Kf8 54. Nb5 Ke8 55. Nc7+ Kd8 56. Kd6 Kc8 57. Ne6 Kb7 (Kb8) 58. Kc5 Ka7 59. Kc6 Ka6 60. Ng5 (Nec5+) Ka7 (Ka5) 61. Nf3 (Ne4) Ka6 62. Nd2 Ka5 63. Kc5 Ka6 64. Nc4 Kb7 65. Kd6 Kc8 66. Na5 Kd8 67. Nb7+ Ke8 68. Ke6 Kf8 69. Nd6 Kg7 70. Kf5 Kh6 71. Kf6 Kh5 72. Nf7 (Ne4) Kg4 73. Ng5 Kh4 74. Kf5 Kg3 75. Ke4 Kg4 76. Nf7 Kh5 (Kg3) 77. Kf5 Kh4 78. Nfe5 Kh5 79. Ng4 Kh4 80. Nf6 Kh3 81. Ke5 Kg3 82. Ke4 Kh3 83. Kf3 Kh4 84. Kf4 Kh3 85. Nh5 (Ne4 Ne8) Kh4 86. Ng3 (Ng7) Kh3 87. Nf5 Kg2 (Kh2) 88. Kg4 Kf1 (Kh1 Kh2 Kg1) 89. Ng3+ Kg2 (Kg1) 90. Ne4 Kf1 91. Kf3 Kg1 92. Nd2 Kh2 93. Nf4 d3 94. Kg4 (Kf2) Kh1 95. Kh3 (Kh4 Kg3) Kg1 96. Kg3 Kh1 97. Nf3 d2 98. Nd3 d1N 99. Kh3 Ne3 (Nb2 Nc3) 100. Nf2\#

\section{$\operatorname{KRP}(d) K B$}

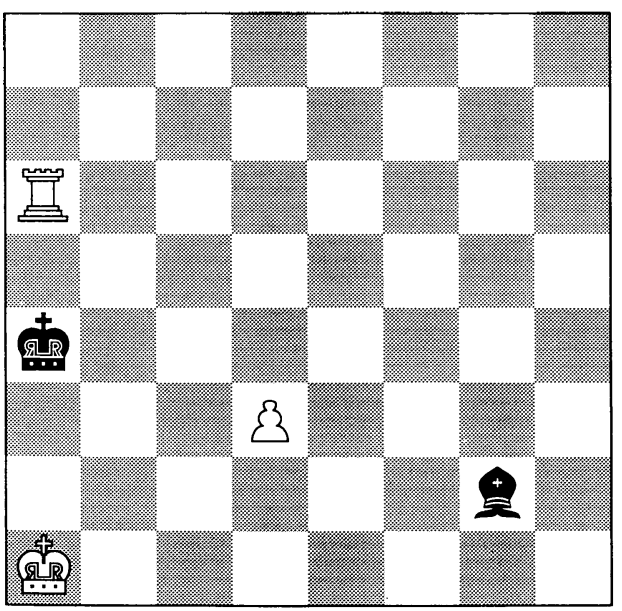

1. ... Kb3 2. Rg6 Bh1 3. Rh6 Bf3 4. Rf6 Bg2 5. Rf2 Bh3 6. Rf3 Bg2 7. Rg3 Bb7 8. Rg7 Ba6 9. d4 Kc4 10. Rg4 Bc8 11. Rf4 Be6 12. Kb2 (Ka2) Kd3 13. Ka3 Kc4 (Kc3) 14. Ka4 Bd7+ (Bc8 Bg8 Bd5) 15. Ka5 Bc6 16. Kb6 Bg2 17. Rg4 Bh1 18. Kc7 Kd5 19. Rg1 Bf3 20. Rf1 Be2 21. Rf4 Bc4 22. Kb6 (Rh4) Bd3 23. Rh4 Bf1 24. Ka5 Kc4 25. Rg4 (Rf4) Bh3 26. Rf4 Bg2 27. Kb6 Bh1 28. Kc7 Kd5 29. Rf1 Bg2 (Be4) 30. Rd1 Be4 31. Kd7 Bf3 32. Rd3 Bg4+ 33. Ke7 Be2 34. Rd2 Bc4 35. $\mathrm{Kd} 7 \mathrm{Bb} 5+(\mathrm{Ba} 6 \mathrm{Bb} 3 \mathrm{Bf} 1)$ 36. Kc7 Ba4 37. Kb6 Be8 38. Ka5 (Ka6) Kc4 39. d5 Kc5 40. d6 Bd7 41. Rd1 Kc6 42. Kb4 Be8 (Be6 Bf5 Bg4 Bh3) 43. Kc3 (Kb3 Rd4) Bd7 44. Rd4 Bf5 45. Kd2 Bc8 46. Rc4+ (Ke3 Ke2) Kd7 47. Rc7+ Kd8 48. Kc3 (Ke3 Kd3) Bd7 (Be6 Bf5 Bg4 Bh3) 49. Kd4 Bg4 (Bh3) 50. Rc2 (Ke5) Bf5 (Be6 Bd1) 51. Rb2 Kd7 (Bh3) 52. Ke5 Bh3 53. Rb7+ (Rb1) Kd8 54. Rb1 Ke8 55. Rh1 Bg4 56. Rh4 Bd7 57. Kd5 Kf7 58. Kc5 (Re4 Rb4) Kg6 59. Kb6 (Rf4 Re4 Rd4 Rb4 Rh2 Rh1) Be8 (Be6 Bf5) 60. Kc7 (Rf4 Re4 Rd4 Rc4 Rb4 Rh2 Rh1) Kg5 61. Rh7 (Rh8 Re4 Rd4 Rc4 Rb4 Rh2 Rh1) Bb5 (Ba4 Bg6 Kf6 Kf4 Kg6 Kf5 Kg4) 62. d7 Ba4 (Bc4 Be2 Bf1 Kf6 Kg6 Kf5 Kg4) 63. d8Q+ Kg6 64. Qd7 (Qd6+ Qd5 Qd2 Kd6 Rg7+ Rf7 Rh6+ Rh3) Bb3 (Bc2) 65. Qd3+ Kf6 (Kg5) 66. Qxb3 Ke5 (Kg5 Kg6 Kf5) 67. Kd7 (Rf7 Rh4 Qf3 Qg3+ Qh3) Kf6 (Kd4 Kf4 Kf5 Ke4) 68. Qd5 (Qb5) Kg6 69. Qh5+ Kf6 70. Rf7\# 
$\operatorname{KQKRP}(\mathbf{c})$

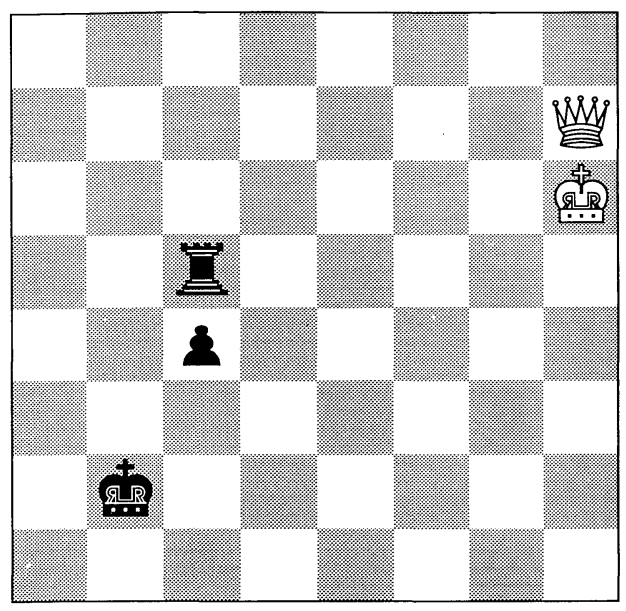

1. Qb7+ Kc1 2. Qh1+ Kb2 3. Qh2+ Kb3 4. Qb8+ Kc2 5. Qd6 Rb5 6. Qc6 Rb4 7. Qe4+ Kb3 8. Qb1+ Kc3 9. Qa2 Rb3 10. Qa4 Rb4 11. Qa3+ Rb3 12. Qc5 Rb4 13. Kg7 Kb3 14. Qd5 Ka3 15. Qc6 (Qe5) Kb3 16. Qe6 Ka3 17. Qe3+ Kb2 18. Qe4 Ra4 19. Qg2+ (Qe2+) Kb3 20. Qd5 Kc2 21. Qd4 Rb4 22. Qe4+ Kb3 23. Qb1+ Kc3 24. Qa2 Rb2 (Kd3) 25. Qa4 Kd3 (Rb4) 26. Qa6 Rc2 (Rd2 Rg2+) 27. Kf6 (Kf7 Kg6) Re2 28. Qb5 Kd4 29. Qa4 Re1 30. Kg5 Kd3 31. Qd7+ Kc3 32. Kf4 Re2 33. Qa4 Kd3 34. Qd1+ Rd2 35. Qb1+ Kd4 36. Kf5 Kc3 37. Ke5 Rb2 38. Qg6 (Qg1) Kb3 39. Qb6+ Ka2 (Kc2) 40. Qa5+ (Qa6+) Kb3 41. Qb5+ Kc3 42. Qa4 Rd2 43. Ke4 (Qa5+ Qa3+) Rd3 44. Qa5+ (Qa3+ Kf4) Kb3 (Kc2) 45. Qb5+ Kc3 46. Qa4 Rd2 47. Qa5+ Kc2 48. Ke3 Rd3+ 49. Ke2 Kb3 50. Qb5+ Kc3 51. Qa4 $\mathrm{Rd} 2+52 . \mathrm{Ke} 1 \mathrm{Rd} 5$ (Rb2) 53. Qa8 Rd3 54. Qa3+ Kd4 55. Qa5 Rb3 56. Kd1 Rb1+ 57. Kc2 Rb3 58. Qf5 (Qg5 Qh5 Kc1) Rc3+ 59. Kb1 (Kb2) Rd3 60. Qa5 (Qg5 Qh5 Ka2 Kb2) Rb3+ (Rd1+) 61. Ka2 Rd3 62. Qb5 Rb3 63. Qc6 Kc3 64. Qd6 (Qc5) Rb2+ 65. Ka3 Rb3+ 66. Ka4 Rb2 67. Qa3+ (Qe5+ Qf6+) Kc2 68. Qe3 c3 69. Qe4+ Kc1 70. Ka3 Kd2 71. Qf3 (Qd4+ Qc4) Rb1 72. Qg2+ (Qf4+ Qf2+) Kd1 73. Qe4 (Qg6) Kc1 74. Qd3 (Qe2) Ra1+ 75. Kb4 (Kb3) c2 (Rb1+) 76. Qe3+ Kb2 (Kb1) 77. Qb3+ Kc1 78. Kc3 Ra3 79. Qxa3+ Kd1 80. Qd6+ (Qa4 Kd3) Kc1 81. Kb3 Kb1 82. Qa3 c1N+ 83. Kc4 (Kc3) Kc2 (Na2) 84. Qd6 Kb1 (Na2 Nb3) 85. Qh2 (Qd2 Qd1) Ka1 (Na2) 86. Kb4 (Qd2 Qc2) Kb1 (Nd3+ Ne2) 87. Qd2 Ka1 (Nd3+ Ne2 Na2+ Nb3) 88. $\mathrm{Ka} 3 \mathrm{~Kb} 1$ (Nd3 Ne2 Na2 Nb3) 89. Qb2\#

\section{KRKBN}

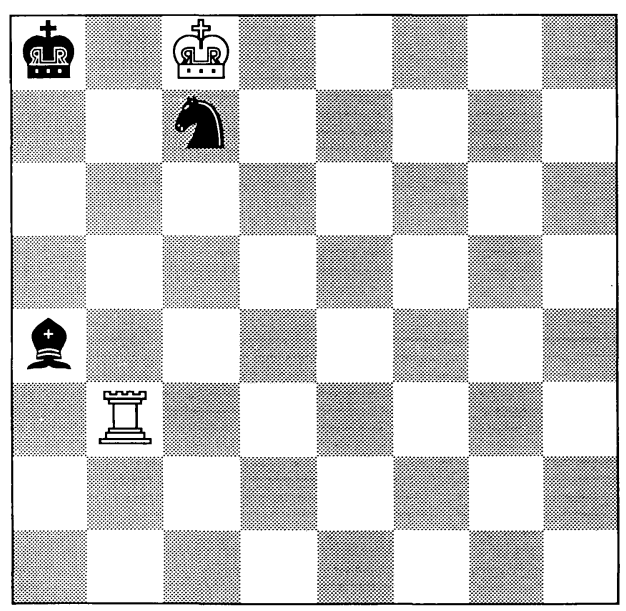

$\operatorname{KRKBP}(\mathbf{a})$

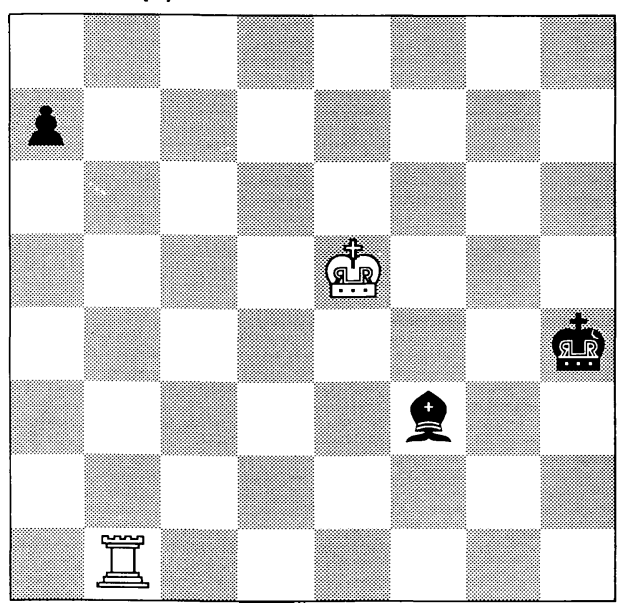

1. Rb6 Nb5 2. Ra6+ Na7+ 3. Kc7 Be8 4. Ra3 Ba4 5. Ra2 Bd1 6. Rb2 Nc8 7. Rg2 Bf3 8. Rg3 (Rg1) Be4 9. Rg5 Bb1 10. Re5 (Ra5+) Bc2 11. Ra5+ Na7 12. Kb6 Kb8 (Bb1) 13. Rxa7 Bb1 14. Ra1 Be4 15. Rc1 (Re1) Bg6 16. Rg1 Bf7 17. Rf1 Be8 18. Rf8 Kc8 19. Rxe8+ Kd7 20. Re5 Kd6 21. Rc5 (Rh5) Ke7 22. Kc7 (Kc6 Rc6) Ke6 23. Rb5 (Ra5) Kf7 (Ke7) 24. Kd6 (Kd7 Rb6) Kf6 25. Kd7 Kg7 (Kf7) 26. Ke6 (Ke7) Kg6 27. Ke7 Kg7 28. Rb6 Kh8 (Kg8) 29. Kf8 (Kf7 Rg6) Kh7 30. Kf7 (Ra6 Rc6 Rd6 Re6 Rf6) Kh8 31. Rh6\#

1. Kf4 Bc6 2. Rc1 Bd5 3. Rd1 Bc6 4. Rd6 Be8 5. Re6 Bf7 6. Re7 Bg6 7. Re1 Kh3 8. Rg1 Bf7 9. Rg3+ Kh2 10. Kf3 a5 11. Kf2 Ba2 12. Ra3 Bb1 13. Ra1 Bg6 14. $\mathrm{Rg} 1 \mathrm{Bc} 2$ (Bh5) 15. Rc1 Ba4 (Be4 Bf5) 16. Rc4 Bd7 17. Rh4+ Bh3 18. Rh5 (Rh6 Rh7 Rh8 Kf3) a4 19. Rh6 (Rh7 Rh8 Rh4) a3 20. Ra6 Bg4 (Bf5) 21. Rxa3 Bd1 22. Rc3 (Rd3 Ra1) Bh5 23. Rc5 (Rd3) Bf7 (Bd1) 24. Rc7 (Rf5) Bg6 25. Rc6 Bh5 26. Rh6 Kh3 27. Rxh5+ $\mathrm{Kg} 4$ 28. Re5 Kf4 29. Re8 (Re3) Kg5 (Kf5 Kg4) 30. Rf8 (Kg3 Kf3) Kg6 (Kg4) 31. Rf4 (Rf3 Kg3 Kf3) Kh5 32. Kf3 Kg5 33. Kg3 Kh6 (Kg6 Kh5) 34. Rg4 Kh7 (Kh5) 35. Kf4 Kh8 (Kh6) 36. Ke5 (Kf5) Kh7 37. Kf6 Kh8 38. Kf7 Kh7 39. Rh4\# 


\section{KRKNP(a)}

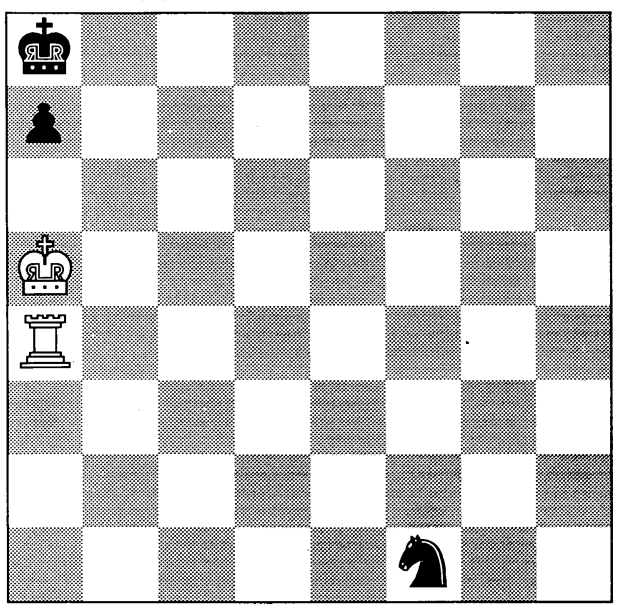

1. Kb4 (Kb5) Kb7 2. Kc5 a5 3. Ra3 Nd2 4. Kd5 Kb6 5. Rd3 Nf1 6. Rb3+ Ka6 7. Kc6 Nd2 8. Rb8 Ka7 9. Rf8 Nc4 10. Kb5 Nd6+ 11. Kc5 Ne4+ 12. Kd4 Nd6 13. Kd5 Nb5 14. Kc5 Nc3 15. Re8 Ka6 16. Re6+ Kb7 17. Re3 Nd1 18. Rb3+ Ka6 19. Kc6 Nf2 20. Rb2 Nd1 (Ne4) 21. Rb6+ (Rb8) Ka7 22. Rb3 Ka6 23. Rd3 Nf2 24. Rd4 (Rd8) Ka7 25. Kb5 Kb7 26. Rd7+ Kc8 27. Kc6 Ng4 28. Ra7 Ne5+ 29. Kd6 Kb8 30. Re7 Nd3 31. $\mathrm{Kc6}$ (Kd5) Nb4+ 32. Kc5 Nd3+ 33. Kb6 Kc8 34. Re4 Nf2 35. Rd4 Nh3 36. Kc6 Ng5 37. Rg4 Ne6 (Nf7) 38. Kd6 (Rg8+) Nd8 39. Rg8 a4 40. Rf8 (Re8 Rh8) a3 41. Ke7 Kb7 (Kb8 Kc7 a2) 42. Rxd8 Ka6 (Kc6 Kb6 a2) 43. Ra8+ (Rd3) Kb5 44. Rxa3 Kb4 45. Ra6 (Ra7 Ra8 Rd3 Re3 Rg3 Rh3 Ra2 Ra1) Kc5 (Kc3 Kb5 Kc4) 46. Ke6 (Rd6 Ra4) Kd4 (Kb5 Kc4) 47. Rd6+ (Ra4+) Kc5 (Kc3 Kc4 Ke4) 48. Rd5+ Kb4 (Kc4) 49. Kd6 (Rd4+) Kc4 50. Kc6 Kb3 (Kb4) 51. Kb5 (Kc5 Rc5) Kc3 52. Rd6 (Rd7 Rd8) Kb2 (Kb3) 53. Kc4 (Kb4) Kc2 54. Kb4 Kb2 55. Rc6 Ka1 (Ka2) 56. Rc2 (Ka3 Kb3) Kb1 57. Kb3 Ka1 58. Rc1\#

1. ... Bd5 2. Rff6 (Rfc3) Ke5 3. Kb7 (Kb8 Ka7 Rfd6 Rg6 Rh6) Bg8 4. Ka6 (Kc7 Kb6 Rb6 Rcd6 Rf8 Rf1) Bb3 (Ba2 Bh7 Kd4 Kd5 Ke4) 5. Kb5 (Kb6 Rcd6) Bg8 (Ba2) 6. Rcd6 (Rf8 Kc5 Kb4) Bb3 (Ba2 Bh7 Ke4) 7. Rf3 (Kc5) Bg8 (Ba2 Bc2) 8. Kc5 Ba2 (Bh7) 9. Rd2 (Rd1) Bb1 10. Re2+ Be4 11. Rxe4+ Kxe4 12. Rf7 (Rf8 Rc3 Rb3 Ra3 Rh3 Rf1) Ke5 (Ke3) 13. Re7+ Kf6 (Kf4 Kf5) 14. Re4 Kg5 (Kg6 Kf5) 15. Kd6 Kf5 16. Rd4 (Rc4 Rb4 Ra4) Kg6 (Kf6) 17. Ke6 (Rd5) Kg5 18. Rc4 (Rb4 Ra4 Re4) Kg6 19. Rc5 (Rg4+) Kg7 20. Rg5+ Kh6 21. Kf6 Kh7 22. Kf7 (Rf5 Re5 Rd5 Rc5 Rb5 Ra5 Rh5+ Rg4 Rg3 Rg2 Rg1) Kh6 23. Rf5 (Re5 Rd5 Rc5 Rb5 Ra5) Kh7 24. Rh5\#

\section{References}

Dekker, S.T., Herik, H.J. van den and Herschberg, I.S. (1987). Complexity Starts at Five. ICCA Iournal, Vol. 10, No. 3, pp. 125-138.

Dekker, S.T., Herik, H.J. van den and Herschberg, I.S. (1990). Perfect Knowledge Revisited. Artificial Intelligence, Vol. 43, No. 1, pp. 111-123.

Herschberg, I.S. and Herik, H.J. van den (1993). Back to Fifty. ICCA Journal, Vol. 16, No. 1, pp. 1-2.

Stiller, L. (1989). Parallel Analysis of Certain Endgames. ICCA Journal, Vol. 12, No. 2, pp. 55-64.

The Editors (1992). Thompson: All about Five Men. ICCA Journal, Vol. 15, No. 3, pp. 140-143. 